Z. Klin. Chem. Klin. Biochem.

13. Jg. 1975, S. 61-67

\title{
Zur Differenzierung gemischter Hyperlipidämien ${ }^{1}$ )
}

\author{
Von G. Schlierf, D. Seidel, U. Claassen und S. Widmann
}

Aus der Medizinischen Universitätsklinik Heidelberg

(Eingegangen am 30. April/2. Oktober 1974)

Ein Vergleich verschiedener Methoden zur Differenzierung gemischter Hyperlipidämien (gleichzeitig Erhöhung von Cholesterin- und Triglyceridkonzentrationen) bei 258 konsekutiven entsprechenden Seren zeigte eine Typenverteilung von: Typ. II $-\mathrm{b}=22 \%$, Typ III $=$ $7 \%$ und Typ IV $=71 \%$. Bei Gesamtcholesterinspiegeln unter 7,25 mmol/1 lag immer ein Typ IV, bei solchen über $8,54 \mathrm{mmol} / 1$ dagegen fast immer (2 \% Ausnahmen) ein Typ II-b oder III vor. Die Berechnung der $\beta$-Lipoproteinkonzen trationen aufgrund der Gesamtcholesterin- und Triglyceridwerte erwies sich bei den Problemfällen (43\%) des vorliegenden Materials mit Cholesterinspiegeln $z$ wischen 7,25 und 8,54 mmol/1 als unzuverlässig. Eine vereinfachte Methode zur Typ III-Diagnostik ohne Anwendung der Ultrazentrifuge zeigte eine sehr gute Übereinstimmung mit der Standardmethode.

\section{Differentiation of mixed hyperlipidaemias}

A comparison of different methods for the differentiation of mixed hyperlipidaemias (concomitant increase in the concentrations of cholesterol and triglycerides) on 258 mixed hyperlipaemic sera showed a type distribution of type II-b $22 \%$, type III $7 \%$, type IV $71 \%$. Total cholesterol concentrations lower than $7.25 \mathrm{mmol} / 1$ were always associated with type IV; above $8.54 \mathrm{mmol} / 1$ total cholesterol, however, the hyperlipaemia was almost always (with $2 \%$ of exceptions) type II-b or III. Calculation of the $\beta$-lipoprotein concentration on the basis of the total cholesterol and trigly ceride values was unreliable in those cases $(43 \%)$ where the cholesterol level was between 7.25 and $8.54 \mathrm{mmol} / \mathrm{l}$. A simplified method for the diagnosis of type III, without the use of the ultracentrifuge, showed good agreement with the standard method.

Die Differenzierung der Hyperlipidämien (Hyperlipoproteinämien) auf der Basis von Lipoproteinmustern $(1,2)$ ist für Belange von Forschung und Klinik in vielen Fällen den früher gebräuchlichen Einteilungsschemen aufgrund der vermehrten Lipidfraktion bzw. aufgrund des Ansprechens auf Testdiäten $(3,4)$ überlegen. Durch die Einführung einfacher lipoproteinelektrophoretischer Verfahren (5-7) gelingt die Differenzierung exogener und endogener Hypertriglyceridämien ohne aufwendige Lipoproteinstudien durch Anfärbung einer Chylomikronen- bzw. verstärkten prä- $\beta$-Lipoproteinbande. Im Zusammenhang mit den Untersuchungen der Fredrickson'schen Arbeitsgruppe zur Validisierung ihres Klassifizierungssystems muß auch die Abgrenzung einer vorher nur vage definierten, meist hereditären Hyperlipoproteinämie mit Vermehrung einer $\beta$-VLDL ${ }^{2}$ )-Fraktion ("floating beta") gesehen werden (Typ-III Hyperlipoproteinämie).

Die Grenzen der Phänotypisierung bzw. der Zuordnung von Hyperlipidämien zu einem der 5 bzw. 6 von Fredrickson et al (8) beschriebenen Lipoproteinmuster ergeben sich in erster Linie durch die Tatsache, daß eine

\footnotetext{
1) Mit Unterstiitzung der Deutschen Forschungsgemeinschaft - SFB 90 -

2) Abkürzungen

VLDL $=$ Very Low Density Lipoproteins

LDL = Low Density Lipoproteins
}

befriedigende quantitative Aussage mit den bisherigen Methoden der Lipoproteinelektrophorese nicht möglich ist. Dies ist besonders schwerwiegend für Hyperlipidämien mit Vermehrung von Triglycerid- und Cholesterinkonzentrationen, bei denen nach Elimination der Fälle mit Vermehrung exogener Triglyceride (Typ I und Typ V) differentialdiagnostisch die Typen II b, III und IV infrage kommen. Hier erlaubt die Lipoproteinelektrophorese allenfalls Vermutungsdiagnosen, die nur durch Einsatz aufwendiger Lipoproteinuntersuchungen (analytische bzw. präparative Ultrazentrifugation) gesichert werden können.

Als Kriterium für die Differentialdiagnose der Typen II und IV gilt die quantitative Bestimmung der $\beta$-Lipoproteine bzw. des $\beta$-Lipoproteincholesterins (2). Bei erhöhtem $\beta$-Lipoproteincholesterin und normalen-Triglyceriden handelt es sich nach den Kriterien von Fredrickson et al um den Typ II-a, bei gleichzeitig erhöhtem Triglyceridspiegel um den Typ II-b, während beim Typ IV $\beta$-Lipoproteine bzw. $\beta$-Lipoproteincholesterin nicht vermehrt sind. Der Nachweis des ,floating-beta" (betaVLDL) bei Typ III nach konventioneller Methodik wird so geführt, daß alle VLDL durch präparative Ultrazentrifugation von den übrigen Lipoproteinen getrennt werden und durch Elektrophorese der Fraktionen der Nachweis des $\beta$-VLDL geführt wird. 
Da die beschriebenen Methoden zur Differenzierung der Typ II b, III und IV Hyperlipoproteinämien unter Einsatz der Ultrazentrifuge nur in wenigen entsprechend ausgerüsteten Laboratorien durchgeführt werden können und teuer und zeitraubend sind, wurden von der Fredrickson'schen Arbeitsgruppe $(9,14)$ Formeln zur rechnerischen Ermittlung des $\beta$-Lipoproteincholesterins aus Gesamttriglyceriden, Gesamtcholesterin und gegebenenfalls $\alpha$-Lipoproteincholesterin angegeben (siehe unten). Die Formeln können nach Angaben der genannten Autoren allerdings nur Anwendung finden, wenn die Gesamttriglyceridkonzentrationen unter $4,56 \mathrm{mmol} / \mathrm{l}^{3}$ ) liegen.

Auch zur Diagnostik des Typs III wurden in den letzten Jahren Methoden beschrieben, die die Anwendung der Ultrazentrifuge überflüssig machen sollen. Es handelt sich dabei

1. um den immunologischen Nachweis des C-Peptids im „floating- $\beta$ ““ $(10)$, also die Diagnostik mittels der Immunelektrophorese und

2. um eine sequentielle Fällung von Lipoproteinen im Elektrophoresegel ohne Verwendung der Ultrazentrifuge (11).

Die vorliegende Arbeit sollte die verschiedenen angegebenen Methoden zur Differenzierung von Hyperlipidämien mit erhöhten Triglycerid- und Cholesterinspiegeln überprüfen und vergleichen.

\section{Material und Methoden}

258 konsekutive Seren aus dem Lipidlabor der Medizinischen Universitätsklinik Heidelberg, bei denen die Triglyceridkonzentration größer als $2,28 \mathrm{mmol} / 1$ und die Cholesterinkonzentration

\footnotetext{
3) Umrechnung: Triglyceride: $\mathrm{mmol} / 1 \times 87,72=\mathrm{mg} / 100 \mathrm{ml}$ Cholesterin: $\mathrm{mmol} / 1 \times 38,67=\mathrm{mg} / 100 \mathrm{ml}$ Triglyceride: $\mathrm{mg} / 100 \mathrm{ml} \times 0,0114=\mathrm{mmol} / 1$ Cholesterin: $\mathrm{mg} / 100 \mathrm{ml} \times 0,0259=\mathrm{mmol} / 1$

Lipidfärbung und Ultrazentrifugation
} Vollserum <d $1.006 \mathrm{~g} / \mathrm{ml}>$ $\oplus$

Typ IV

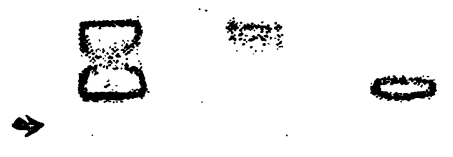

Typ III

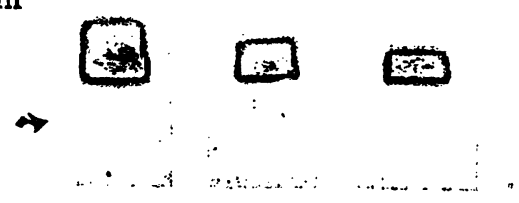

größer als 6,48 mmol/1 war, wurden untersucht. Bei allen Seren fand folgende Methodik Anwendung:

a) Bestimmung der Gesamttriglyzeride im Autoanalyzer ${ }^{4}$ (12)

b) Bestimmung des Gesamtcholesterins im Autoanalyzer ${ }^{4}$ ) (Methode N-24 a)

c) Bestimmung des $\beta$-Lipoproteincholesterins nach Ultrazentrifugation und Lipoproteinpräzipitation (2). Als Grenzwerte für $\beta$-Lipoproteincholesterin gelangten nach den Angaben von Fredrickson folgende Werte zur Anwendung:

$\begin{array}{cc}\text { Alter (Jahre) } & \mathrm{mmol} / \mathrm{l} \\ & \cdot 1 \\ 0-19 & 4,40 \\ 20-29 & 4,40 \\ 30-39 & 4,92 \\ 40-49 & 4,92 \\ >50 & 5,44\end{array}$

Bei fehlender Altersangabe wurde ein Grenzwert von $4,92 \mathrm{mmol} / 1$ angenommen.

d) „konventionelle“ Typ-III-Diagnostik (2)

e) immunologische Typ-III-Diagnostik (10) (Abb. 1) Das Muster der Typ III-Hyperlipoproteinämie ist gekennzeichnet durch das Auftreten eines Plasmalipoproteins mit abnormen physiko-chemischen Eigenschaften (VLDL mit $\beta$-Mobilität; $\beta$-VLDL), dessen Nachweis beweisend für diese . Form von Fettstoffwechselstörung ist.

Bei der Trennung des Serums in die Dichteklasse $d>$ und $<1,006 \mathrm{~g} / \mathrm{ml}$ durch die Ultrazentrifuge mit nachfolgender Lipidelektrophorese beider Fraktionen zeigt die Typ III Hyperlipoproteinämie im Unterschied zu allen anderen Formen in der VLDL-Klasse nicht nur prae- $\beta$-Lipoproteine, sondern die abnorme und charakteristische Komponente, die $\beta$-Mobilität entwickelt (2).

Neben seinen abnormen physiko-chemischen Eigenschaften unterscheidet sich das für Typ III charakteristische $\beta$-VLDL

4) Qualitätskontrolle für Cholesterin

1 , Soll $4,40 \mathrm{mmol} / 1 \bar{x}$ von $n=20: 4,44 \mathrm{mmol} / 1$ Variationskoeffizient $2,9 \%$

2 , Soll $8,73 \mathrm{mmol} / 1 \bar{x}$ von $\mathrm{n}=20: 8,47 \mathrm{mmol} / 1$ Variationskoeffizient $3,3 \%$

Qualitätskontrolle für Triglyceride

3 , Soll $3,77 \mathrm{mmol} / 1 \overline{\mathrm{x}}$ von $\mathrm{n}=38: 3,43 \mathrm{mmol} / 1$ Variationskoeffizient $4,9 \%$

Immun elektrophorese Präzipitationstechnik mit Lösung 1 mit Lösung 2
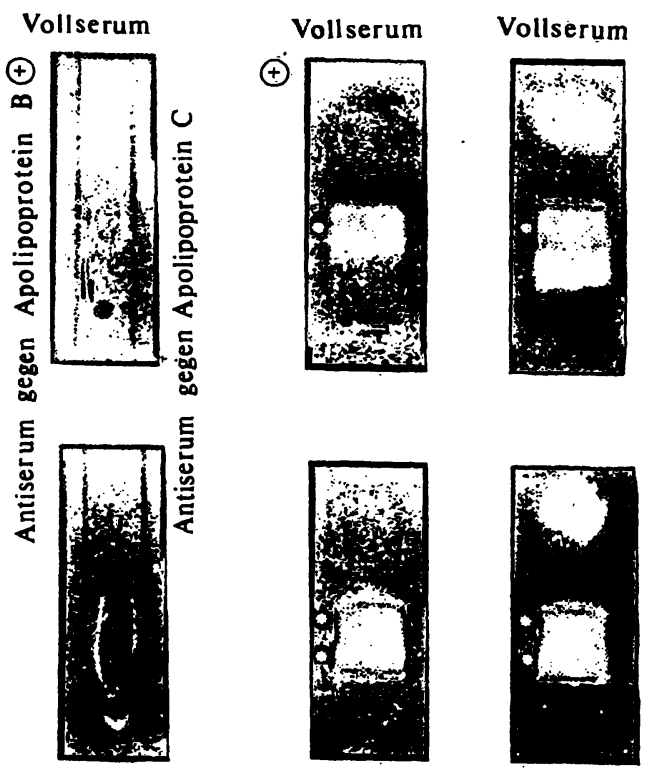

Abb. 1. Verfahren zur Diagnostik der Typ III-Hyperlipoproteinämie nach Wieland und Seidel (11). 
auch hinsichtlich seines Proteinanteils von normalen $\beta$-Lipo proteinen. Es zeigt neben dem Proteinanteil normaler $\beta$ Lipoproteine, Apolipoprotein B, auch den hauptsächlichen Proteinanteil der VLDL-Fraktion, Apolipoprotein C. Diese Eigenschaft ermöglicht seinen Nachweis durch immunologische Techniken ohne Verwendung der Ultrazentrifuge.

Testet man Vollserum in der Immunelektrophorese mit gegen Apolipoprotein B und $\mathbf{C}$ gerichtetem Antiserum, wird der Unterschied zwischen den Typen deutlich. Während alle anderen Formen von Hyperlipoproteinämie (in der $\mathbf{A b b}$. Beispiel eines Typ IV-Hyperlipoproteinämie-Musters) nur in der prae- $\beta$-Lipoproteinbande mit anti-Lipoprotein C-Serum reagieren, zeigt die Typ III-Hyperlipoproteinämie sowohl in der $\beta$ - wie in der prae- $\beta$-Position diese Reaktion. Da sich das Apolipoprotein B sowohl in der prae- $\beta$ - wie in der $\beta$-Bande findet, eignet sich das Antiserum gegen Apolipoprotein B in diesem Test als Referenz zur Festlegung der $\beta$ - bzw. prae- $\beta$-Position.

f) Präzipitationsmethode zur Diagnose des Typ III (11) (Abb. 1).

Eine zweite und zuverlässige Methode zur Diagnose oder zum Ausschluß eines Typ III-Hyperlipoproteinmusters ohne Verwendung der Ultrazentrifuge stellt die stufenweise Präzipitationstechnik der Lipoproteine dar.

Hierbei werden nach dem Elektrophoreselauf zunächst selektiv die Lipoproteine der VLDL- $(\mathrm{d}<1,006 \mathrm{~g} / \mathrm{ml})$ Fraktion durch Präzipitation einer $0,1 \mathrm{~mol} / 1 \mathrm{MgCl}_{2}$-Lösung, dic $0,15 \%$ Natrium-Heparin und $1,0 \% \mathrm{NaCl}$ enthält (Lösung 1), zur Darstellung gebracht, die Position der auftretenden Banden markiert und in einer zweiten Präzipitationsstufe durch eine $0,2 \mathrm{~mol} / 1$ Calciumchloridlösung, die $0,6 \% \mathrm{Na}$-Dextransulfat 500 enthält (Lösung 2), alle übrigen Lipoproteinbanden auf derselben Platte dargestellt. Die Markierung zeigt jetzt an, ob die VLDL-Fraktion, die für das Typ III-Hyperlipoproteinämiemuster charakteristische $\beta$-VLDL-Komponente enthält oder nicht.

Sowohl für die immunologische sowie für die Präzipitationstechnik ist der Ausschluß von sekundären Dy slipoproteinämien, speziell solcher, die im Verlauf von Lebererkrankungen auftreten können, erforderlich, eine Forderung, die generell für die Diagnostik der verschiedenen Formen von Hyperlipoproteinämie gilt.

Bei Lebererkrankungen finden sich VLDL in der $\beta$-Position (13). Hier können bei den beschriebenen Methoden e) und f) ebenfalls Typ-III-Muster resultieren.

Mit der durch die Methoden a)-d) durchgeführten Klassifizierung (Standardmethoden) wurde sowohl die von Friedewald et al (14) angegebene Formel zur $\beta$-Lipoproteincholesterin-Bestimmung (angepaßt für die Konzentrationsangaben in $\mathrm{mmol} / \mathrm{l}$ )

$\beta$-Lipoproteincholesterin $=$ Gesamtcholesterin -

$\left[\frac{\text { Triglyceride }}{2,2}+\alpha\right.$-Lipoproteincholesterin $]$

(Formel I)

als auch die vereinfachte Formel von Fredrickson et al (2):

$\beta$-Lipoproteincholesterin $=$ Gesam tcholesterin

$\left[\frac{\text { Triglyceride }}{2,2}+1,17\right]$

(Formel II)

verglichen. Ferner wurde versucht, für bestimmte Konstellationen von Gesamttriglycerid- und Gesamtcholesterinwerten die Wahrscheinlichkeit für die Zugehörigkeit der entsprechenden Seren zu den nach Referenzmethoden a-d ermittelten Hyperlipoproteinämietypen aufzuzeigen.

\section{Ergebnisse}

Aufgrund der konventionellen, detaillierten Lipidanalytik unter Verwendung der Ultrazentrifuge (Verfahren a) -d) konnten von den 258 untersuchten Seren $185 \mathrm{dem}$ Typ IV, 56 dem Typ II-b und 17 dem Typ III zugeordnet werden. Die Ergebnisse der Laboranalysen sind in Tabelle 1 zusammengestellt.

Abbildung 2 zeigt die Verteilung der Triglycerid- und Cholesterinkonzentrationen bei den Patienten mit Typ II-b. Der Bereich der Triglyceridspiegel reichte von 2,28-7,75 mmol/1, Mittelwert 4,0 mmol/1. Bei den Cholesterinspiegeln war der Bereich 7,25-16,73 mmol/1 Mittelwert $9,71 \mathrm{mmol} / 1$.

Abbildung 3 zeigt die Verteilung der Triglycerid- und Cholesterinkonzentrationen der Patienten mit Typ III. Hier war der Bereich für Triglyceride und Cholesterin und die entsprechenden Mittelwerte: Triglyceride 3,7338,76, Mittelwert 12,68 mmol/1, Cholesterin 7,51-17,09, Mittelwert 9,97 mmol/1.

Abbildung $4 \mathrm{a}$ und $4 \mathrm{~b}$ zeigen Triglycerid- und Cholesterinkonzentrationen der Patienten mit Typ IV.

Bereich für Triglyceride: $2,28-57 \mathrm{mmol} / 1$, Mittelwert 7,3 mmol/1, Bereich für Cholesterin: $6,48-10,36 \mathrm{mmol} / 1$, Mittelwert 8,08 mmol/1.

\section{Diskussion}

\section{Typ II-b-Hyperlipoproteinämie}

Der Anteil von Seren mit Typ II-b-Muster unter 258 unausgewählten konsekutiven Proben mit Erhöhung der Triglyceride und des Cholesterins beträgt 21,7\%. Dabei lag der Gesamtcholesterinspiegel bei keinem dieser Seren unter 7,25 mmol/1. Das Vorliegen einer Typ-II-bHyperlipoproteinämie bei einem Gesamtcholesterin unter $7,25 \mathrm{mmol} / \mathrm{l} \mathrm{muß} \mathrm{deshalb} \mathrm{als} \mathrm{selten} \mathrm{angesehen} \mathrm{werden;}$ bei Erwachsenen scheinen $\beta$-Lipoproteincholesterin-Bestimmungen bei einem Serumcholesterinwert unter $7,25 \mathrm{mmol} / \mathrm{l}$ nicht notwendig zu sein.

Die Diagnostik der Typ II-b-Hyperlipoproteinämie mit der Formel II erscheint problematisch. Abgesehen von den Fällen mit Triglyceriden $>4,56 \mathrm{mmol} / \mathrm{l}$ ( 10 von 56) wo die Formel nicht anwendbar sein soll (9), entstehen Fehler durch die relativ große Streuung des $\alpha$ Lipoproteincholesterins und durch die Berechnung der VLDL-Triglyceride aus der Gesamttriglyceridkonzentration, die einen fixen Triglyceridanteil der VLDLLipoproteine voraussetzt. Offensichtlich ist diese Voraussetzung sehr häufig nicht gegeben $(15,16)$. Im vorliegenden Material wären bei Anwendung dieser Formel von den 56 Patienten mit Typ II-b 10 (17,9\%) dem Typ IV zugeordnet worden. Unter Verwendung der Formel I, bei der die gemessenen Werte für $\alpha$-Lipoproteincholesterin eingesetzt werden, ist die Trefferquote besser: von den 46 auswertbaren Seren wären 5 dem Typ IV zugeordnet worden. 
Tab. 1. Werte für Gesamttrigly ceride, Gesamtcholesterin, $\beta$-Lipoproteincholesterin und $\alpha$-Lipoproteincholesterin sowie berechnete Werte für $\beta$-Lipoproteincholesterin nach den Formeln I und II.

*) Patient würde aufgrund der berechneten Werte als Typ IV klassifiziert

**) Patient würde aufgrund der berechneten Werte als Typ II-b klassifiziert.

Typ II-b

\begin{tabular}{lllll}
\hline Trigly- & Chole- & $\beta$-Lipo- & $\alpha$-Lipo- & Alter \\
ceride & sterin & $\begin{array}{l}\text { berechnetes } \\
\text { protein- } \\
\text { chole- } \\
\text { protein- }\end{array}$ & $\begin{array}{l}\beta \text {-Lipoprotein- } \\
\text { chole- } \\
\text { sterin }\end{array}$ &
\end{tabular}

$[\mathrm{mmol} / \mathrm{l}][\mathrm{mmol} / \mathrm{l}][\mathrm{mmol} / \mathrm{l}][\mathrm{mmol} / \mathrm{l}][\mathrm{Jahre}][\mathrm{mmol} / \mathrm{l}]$ nach Formel

I II

\begin{tabular}{|c|c|c|c|c|c|c|}
\hline 4,56 & 9,32 & 6,92 & 0,62 & 69 & & \\
\hline 3,76 & 9,38 & 7,51 & 0,73 & & 6,94 & 6,50 \\
\hline 3,53 & 9,63 & 5,65 & 0,88 & 52 & 7,15 & 6,60 \\
\hline 2,57 & 8,50 & 6,29 & 0,85 & 31 & 6,48 & 6,16 \\
\hline 3,93 & 7,67 & 5,67 & 0,78 & 59 & $5,10^{*}$ & $4,71 *$ \\
\hline 2,74 & 7,77 & 6,06 & 0,78 & 53 & 5,75 & $5,36^{*}$ \\
\hline 2,71 & 7,41 & 5,44 & 1,3 & & $4,87^{*}$ & $.5,00^{*}$ \\
\hline 3,26 & 7,51 & 5,52 & 0,78 & 49 & 5,26 & 4,87 \\
\hline 5,53 & 10,05 & 6,40 & 1,22 & 40 & & \\
\hline 3,36 & 7,30 & 4,97 & 1,17 & 42 & $4,61^{*}$ & $4,61^{*}$ \\
\hline 2,28 & 16,73 & 10,88 & 0,78 & 41 & 14,92 & 14,53 \\
\hline 2,51 & 9,07 & 6,94 & 0,91 & 46 & 7,02 & 6,76 \\
\hline 2,68 & 8,29 & 5,65 & 1,61 & 48 & 5,46 & 5,70 \\
\hline 2,62 & 9,22 & 6,48 & 1,81 & 53 & 6,22 & 6,86 \\
\hline 2,68 & 10,10 & 7,30 & 1,55 & 45 & 7,33 & 7,72 \\
\hline 2,79 & 8,55 & 5,49 & 1,65 & 72 & 5,67 & 6,11 \\
\hline 2,85 & 7,56 & 5,13 & 2,25 & 45 & $4,01 *$ & 5,10 \\
\hline 2,51 & 8,34 & 5,44 & 1,3 & 39 & 5,91 & 6,03 \\
\hline 6,84 & 10,15 & 5,39 & 1,81 & 43 & & \\
\hline 3,42 & 8,81 & 5,49 & 1,3 & 30 & 5,96 & 6,09 \\
\hline 2,68 & 7,77 & 5,70 & 0,91 & 69 & 5,65 & $5,39 *$ \\
\hline 4,73 & 8,86 & 6,06 & 0,93 & 58 & & \\
\hline 3,42 & 7,46 & 5,39 & 0,57 & 49 & 5,34 & $4,74^{*}$ \\
\hline 2,45 & 7,77 & 5,54 & 0,91 & 52 & 5,75 & 5,49 \\
\hline 2,57 & 7,51 & 5,52 & 0,65 & 52 & 5,70 & $5,18^{*}$ \\
\hline 3,19 & 8,60 & 5,70 & 0,83 & 47 & 6,32 & 5,98 \\
\hline 3,08 & 7,77 & 7,02 & 0,65 & & 5,72 & 5,20 \\
\hline 2,28 & 9,84 & 5,88 & 2,67 & 52 & 6,14 & 7,64 \\
\hline 3,93 & 8,70 & 6,22 & 1,3 & 47 & 5,62 & 5,75 \\
\hline 3,76 & 7,51 & 5,18 & 0,78 & & 5,02 & 4,64 \\
\hline 3,31 & 7,25 & 4,45 & 0,88 & 29 & 4,87 & 4,58 \\
\hline 7,07 & 8,86 & 5,96 & 1,04 & 23 & & \\
\hline 2,28 & 8,29 & 5,21 & 1,55 & 49 & 5,70 & 6,09 \\
\hline 2,74 & 12,54 & 9,61 & 0,91 & 55 & 10,39 & 10,13 \\
\hline 7,75 & 8,96 & 5,26 & 1,22 & 58 & & \\
\hline 2,79 & 9,51 & 7,51 & 1,17 & 61 & 7,07 & 7,07 \\
\hline 2,79 & 12,95 & 9,79 & 1,35 & 60 & 10,33 & 10,52 \\
\hline 3,36 & 11,97 & 9,95 & 0,93 & 29 & 9,51 & 9,27 \\
\hline 6,84 & 8,24 & 6,37 & 0,31 & 58 & & \\
\hline 2,92 & 8,13 & 5,65 & 1,04 & 54 & 5,78 & 5,91 \\
\hline 7,98 & 9,07 & 6,11 & 1,55 & & & \\
\hline 3,71 & 7,98 & 5,44 & 0,98 & 48 & 5,31 & 5,13 \\
\hline 3,93 & 8,03 & 5,26 & 1,04 & 46 & 5,21 & 5,08 \\
\hline 2,96 & 8,39 & 6,68 & 0,78 & 67 & 6,27 & 5,88 \\
\hline 2,74 & 8,29 & 6,55 & 0,91 & 54 & 6,14 & 5,88 \\
\hline 10,83 & 9,32 & 6,16 & 1,04 & 62 & & \\
\hline 2,45 & 11,81 & 7,93 & 2,18 & 52 & 8,52 & 9,53 \\
\hline 2,85 & 8,81 & 6,37 & 1,40 & 66 & 6,11 & 6,35 \\
\hline 3,53 & 7,67 & 5,75 & 1,09 & 57 & $4,97 *$ & 4,89 \\
\hline 2,37 & 8,18 & 5,83 & 1,17 & & 5,80 & 5,80 \\
\hline 3,19 & 7,61 & 6,00 & 0,88 & 47 & 5,85 & 5,57 \\
\hline 3,14 & 8,91 & 4,53 & 1,24 & 29 & 4,95 & 5,02 \\
\hline 2,79 & & 6,53 & 1,17 & 58 & 6,48 & 6,48 \\
\hline 3,36 & 8,34 & 5,57 & 1,01 & 51 & 5,80 & 5,65 \\
\hline 5,76 & 7,25 & 5,57 & 0,65 & & & \\
\hline 3,31 & 7,64 & 5,44 & 0,78 & 61 & 5,36 & 4,97 \\
\hline
\end{tabular}

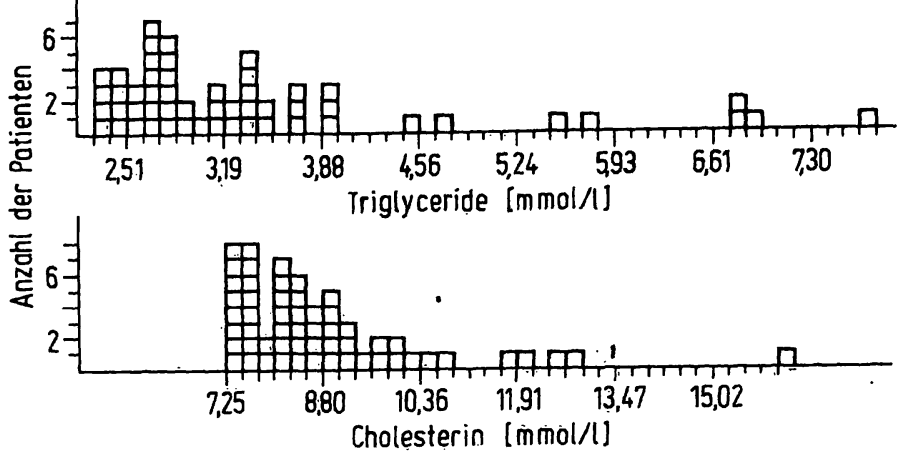

Abb. 2. Verteilung der Triglycerid- und Cholesterinkonzentrationen bei Typ IIb-Hyperlipoproteinämie.

\section{Typ III-Hyperlipoproteinämie}

Von 258 konsekutiven Seren mit Erhöhung der Triglycerid- und Cholesterinkonzentrationen konnten 17 $(6,6 \%)$ als Typ III identifiziert werden. Wie Abbildung 2 zeigt, wurde ein Cholesterinspiegel unter $7,51 \mathrm{mmol} / 1$ nicht beobachtet und macht das Vorliegen einer TypIII-Hyperlipoproteinämie unwahrscheinlich. Ein suspektes Lipoproteinmuster bei der Elektrophorese vor Ultrazentrifugation (verbreiterte $\beta$-Bande) ließ sich nur bei 9 Patienten mit Typ III Hyperlipoproteinämie nachweisen. Die Übereinstimmung der drei zur Typ IIII-Diagnostik verwendeten Methoden war bis auf eine Ausnahme vollständig. Es handelte sich um ein mit der Post versandtes Serum mit alterierter Mobilität der Lipoproteinbanden, bei dem der Typ III durch die Methode e) und f), nicht jedoch durch Methode d) diagnostiziert wurde. Wegen des geringen methodischen Aufwandes und der Möglichkeit der Typ III-Diagnostik ohne Verwendung der Ultrazentrifuge erscheint die Präzipitationstechnik als wichtige Bereicherung der Methoden zur Erstellung eines Lipidstatus.

Typ III

\begin{tabular}{rrll}
\hline $\begin{array}{l}\text { Triglyceride } \\
\text { [mmol/1] }\end{array}$ & $\begin{array}{l}\text { Cholesterin } \\
\text { [mmol/1] }\end{array}$ & $\begin{array}{l}\beta \text {-Lipoprotein- } \\
\text { cholesterin } \\
\text { [mmol/1] }\end{array}$ & $\begin{array}{l}\alpha \text {-Lipoprotein- } \\
\text { cholesterin } \\
\text { [mmol/1] }\end{array}$ \\
\hline 12,54 & 7,77 & 2,90 & 0,88 \\
21,66 & 14,25 & 4,51 & 0,52 \\
3,82 & 8,29 & 2,90 & 0,73 \\
4,10 & 9,07 & 2,49 & 2,43 \\
6,04 & 8,29 & 4,64 & 0,65 \\
5,30 & 11,40 & 5,70 & 0,75 \\
38,19 & 7,98 & 2,85 & 1,04 \\
11,40 & 17,09 & 5,59 & 0,85 \\
4,56 & 7,77 & 2,67 & 1,06 \\
4,67 & 8,29 & 3,19 & 0,70 \\
35,34 & 8,81 & 2,33 & 0,62 \\
14,82 & 9,27 & 2,82 & 1,17 \\
6,50 & 7,77 & 2,90 & 0,78 \\
5,02 & 8,81 & 2,98 & 0,90 \\
5,70 & 7,30 & 2,64 & 1,04 \\
4,05 & 7,72 & 3,11 & 0,75 \\
3,59 & 8,44 & 5,18 & 0,83 \\
\hline
\end{tabular}




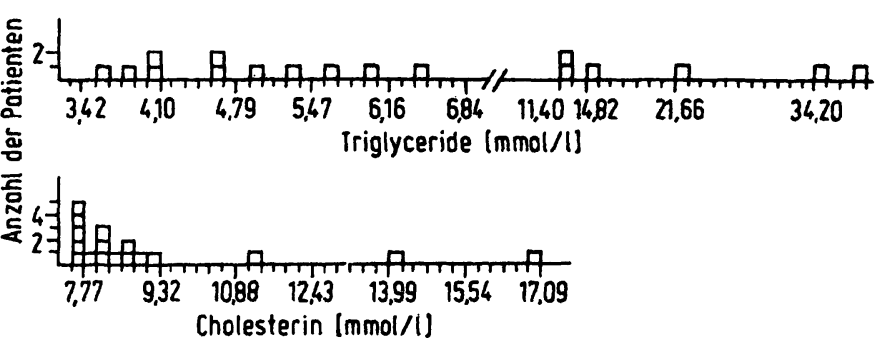

Abb. 3. Verteilung der Trigly cerid- und Cholesterinkonzentrationen bei Typ III-Hyperlipoproteinämie.

\section{Typ IV-Hyperlipoproteinämie}

Von 258 Seren mit erhöhten Triglycerid- und Cholesterinkonzentrationen konnten $185(71,7 \%)$ aufgrund normaler $\beta$-Lipoproteincholesterinkonzentration als Typ IV angesprochen werden. Die Inspektion von Abbildung 3 zeigt, daß bei einem Gesamtcholesterinspiegel über $8,55 \mathrm{mmol} / 1$ nur noch bei $2 \%$ mit dem Vorliegen eines Typ IV gerechnet werden muß.

Unter Anwendung der Formeln I bzw. II zur Berechnung der $\beta$-Lipoproteinkonzentrationen würden von 185 Patienten mit Typ IV-Muster 6 bzw. 7 als Typ II-b- klassifiziert.

Während aus den geschilderten Gründen bei ,gemischten Hyperlipidämien" aus der Kenntnis der Gesamtcholesterinspiegel ohne weitere Lipoproteinanalysen eine relativ sichere Typenzuordnung bei Gesamtcholesterinwerten über $8,55 \mathrm{mmol} / 1$ (mit großer Wahrscheinlichkeit Typ II-b oder III) bzw. unter 7,25 mmol/1 (mit großer Wahrscheinlichkeit Typ IV) möglich ist, sind in den Fällen unseres Materials (immerhin $43 \%$ ), bei denen neben erhöhten Triglyceridwerten Gesamtcholesterinspiegel zwischen 7,25 und $8,55 \mathrm{mmol} / 1$ bestanden, aus den Lipidwerten keine Aussagen bezüglich der Typen II-b, III oder IV möglich. Nach Ausschluß der Fälle mit Typ III durch die Methode der stufenweisen Lipoproteinpräzipitation im Agarosegel nach Wieland und Seidel (11) muß hier zur sicheren Differenzierung zwischen Typ II-b und IV eine Messung des $\beta$ - $\left.(\mathrm{LDL})^{2}\right)$ Cholesterins erfolgen. Eine Verwendung der Formel I ergäbe bei diesen Typ II-b Patienten (Cholesterinspiegel zwischen 7,25 und $8,55 \mathrm{mmol} / \mathrm{l}$ ) eine Trefferquote von nur $62 \%$, bei Typ IV-Patienten eine Trefferquote von $85 \%$.

$\mathrm{Da}$ es möglich ist, durch Doppelpräzipitation den Ultrazentrifugenschritt zu umgehen und trotzdem akkurate LDL-Konzentrationen zu erhalten, zeigt eine Arbeit von Wilson und Spiger (17), die neben den wesentlich niedrigeren Instrumentierungskosten auch den Zeitaufwand für quantitative Lipoproteinbestimmungen wesentlich verkürzt.
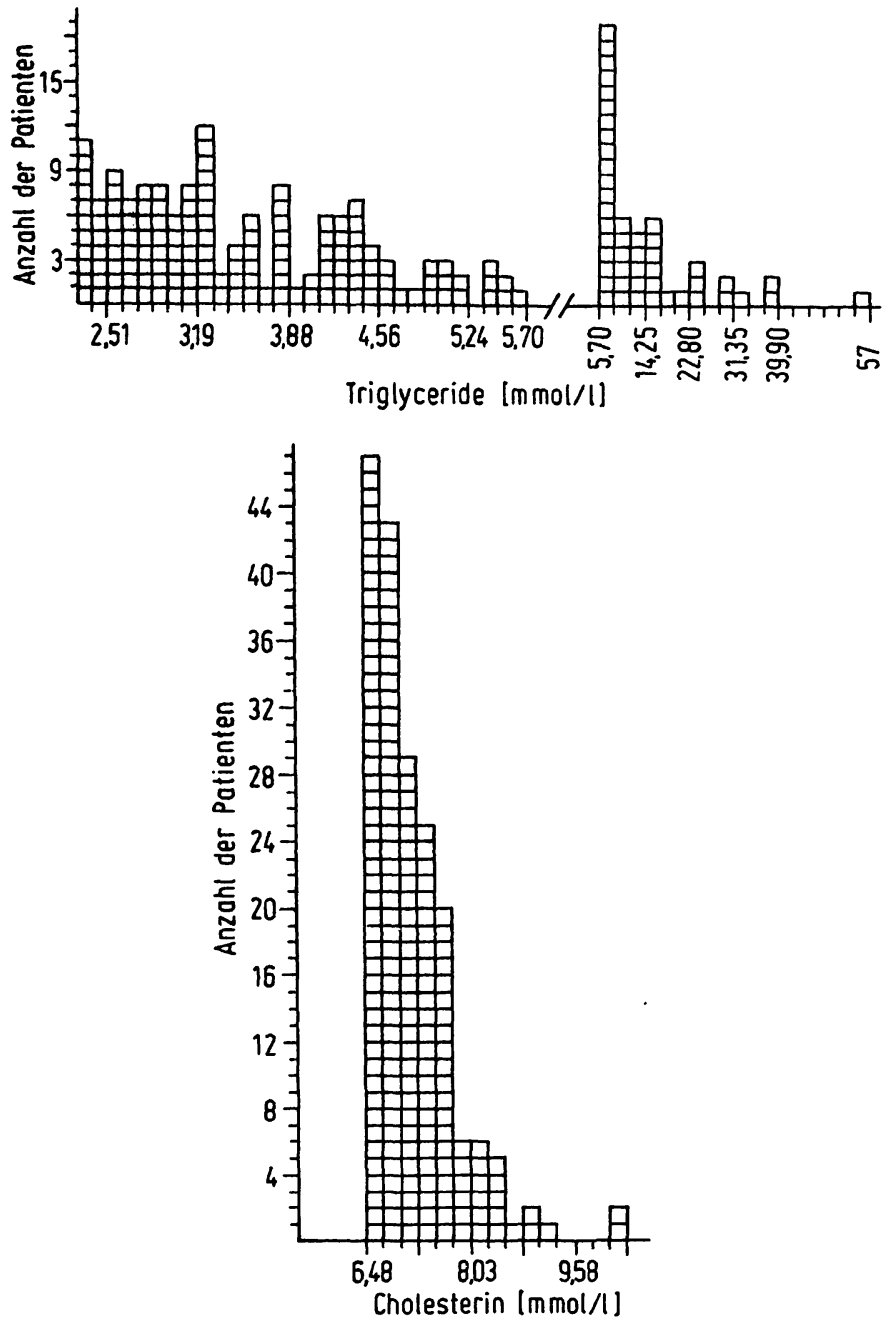

Abb. 4. Verteilung der Triglycerid-(a) und Cholesterinkonzentrationen (b) bei Typ IV-Hy perlipoproteinämie.

Typ IV

\begin{tabular}{|c|c|c|c|c|c|c|}
\hline \multirow{3}{*}{$\begin{array}{l}\text { Trigly- } \\
\text { ceride }\end{array}$} & \multirow{3}{*}{$\begin{array}{l}\text { Chole- } \\
\text { sterin }\end{array}$} & \multirow{3}{*}{$\begin{array}{l}\beta \text {-Lipo- } \\
\text { protein- } \\
\text { chole- } \\
\text { sterin } \\
\text { [mmol/1] }\end{array}$} & \multirow{3}{*}{$\begin{array}{l}\alpha \text {-Lipo- } \\
\text { protein- } \\
\text { chole- } \\
\text { sterin } \\
{[\mathrm{mmol} / \mathrm{l}]}\end{array}$} & Alter & \multicolumn{2}{|c|}{$\begin{array}{l}\text { berechnetes } \\
\beta \text {-Lipoprotein- } \\
\text { cholesterin }\end{array}$} \\
\hline & & & & \multicolumn{3}{|c|}{$\begin{array}{r}\text { [Jahre] [mmol/1] } \\
\text { nach Formel }\end{array}$} \\
\hline & & & & & I & II \\
\hline 30,21 & 8,24 & 3,42 & 0,73 & \multicolumn{3}{|l|}{48} \\
\hline 22,80 & 6,68 & 4,20 & 0,65 & & & \\
\hline 5,70 & 8,65 & 5,00 & 0,52 & \multicolumn{3}{|l|}{50} \\
\hline 10,60 & 7,25 & 2,20 & 0,70 & \multicolumn{3}{|l|}{62} \\
\hline 9,52 & 8,44 & 4,35 & 1,22 & \multirow{2}{*}{\multicolumn{3}{|c|}{$\begin{array}{l}58 \\
58\end{array}$}} \\
\hline 6,84 & 8,03 & 4,79 & 1,30 & & & \\
\hline 2,79 & 6,58 & 3,16 & 1,17 & 69 & \multirow{3}{*}{$\begin{array}{l}4,14 \\
3,32\end{array}$} & \multirow{3}{*}{$\begin{array}{l}4,14 \\
5,72 * *\end{array}$} \\
\hline 2,74 & 8,13 & 4,35 & 3,57 & 53 & & \\
\hline 13,68 & 8,39 & 4,01 & 0,39 & 33 & & \\
\hline 2,51 & 6,73 & 3,89 & 0,78 & 61 & 4,82 & 4,43 \\
\hline 2,57 & 6,89 & 4,58 & 0,93 & 55 & 4,79 & 4,56 \\
\hline 2,35 & 7,36 & 4,97 & 1,40 & 56 & 4,87 & 5,10 \\
\hline 3,31 & 6,99 & 4,56 & 0,73 & 52 & 4,77 & 4,33 \\
\hline 3,82 & 6,76 & 3,57 & 2,25 & 38 & 2,77 & 3,86 \\
\hline 4,22 & 8,03 & 5,05 & 0,78 & 75 & 5,34 & 4,95 \\
\hline 2,91 & 6,99 & 3,16 & 1,04 & 67 & 4,64 & 4,51 \\
\hline 2,96 & 6,84 & 3,39 & 0,85 & 49 & 4,64 & 4,33 \\
\hline 4,22 & 7,25 & 4,66 & 1,17 & 54 & 4,17 & 4,17 \\
\hline 2,85 & 6,99 & 4,35 & 1,55 & 64 & 4,14 & 4,53 \\
\hline 3,59 & 8,18 & 5,18 & 1,50 & 62 & 5,05 & 5,39 \\
\hline 3,36 & 6,68 & 4,35 & 0,78 & 30 & 4,38 & 3,99 \\
\hline
\end{tabular}


Typ IV

\begin{tabular}{|c|c|c|c|c|c|}
\hline $\begin{array}{l}\text { Trigly- } \\
\text { ceride }\end{array}$ & $\begin{array}{l}\text { Chole- } \\
\text { sterin }\end{array}$ & $\begin{array}{l}\text { B-Lipo- } \\
\text { protein- } \\
\text { chole- } \\
\text { sterin }\end{array}$ & $\begin{array}{l}\alpha \text {-lipo- } \\
\text { protein- } \\
\text { chole- } \\
\text { sterin }\end{array}$ & Alter & $\begin{array}{l}\text { bercchnetes } \\
\beta \text {-Lipoprotein- } \\
\text { cholesterin }\end{array}$ \\
\hline
\end{tabular}

[inmol/l] [mmol/l] [mmol/1] [mmol/l] [Jahre] [mmol/l]
Typ IV

Trigly- Chole- BLipo- $\alpha$-Lipo- Alter berechnetes

ceride sterin protein- protein- B-Lipoproteinchole- chole- cholesterin

sterin sterin

[mmol/1] (mmol/1] [mmol/l] [mmol/l] [Jahre] [mmol/1] nach forme

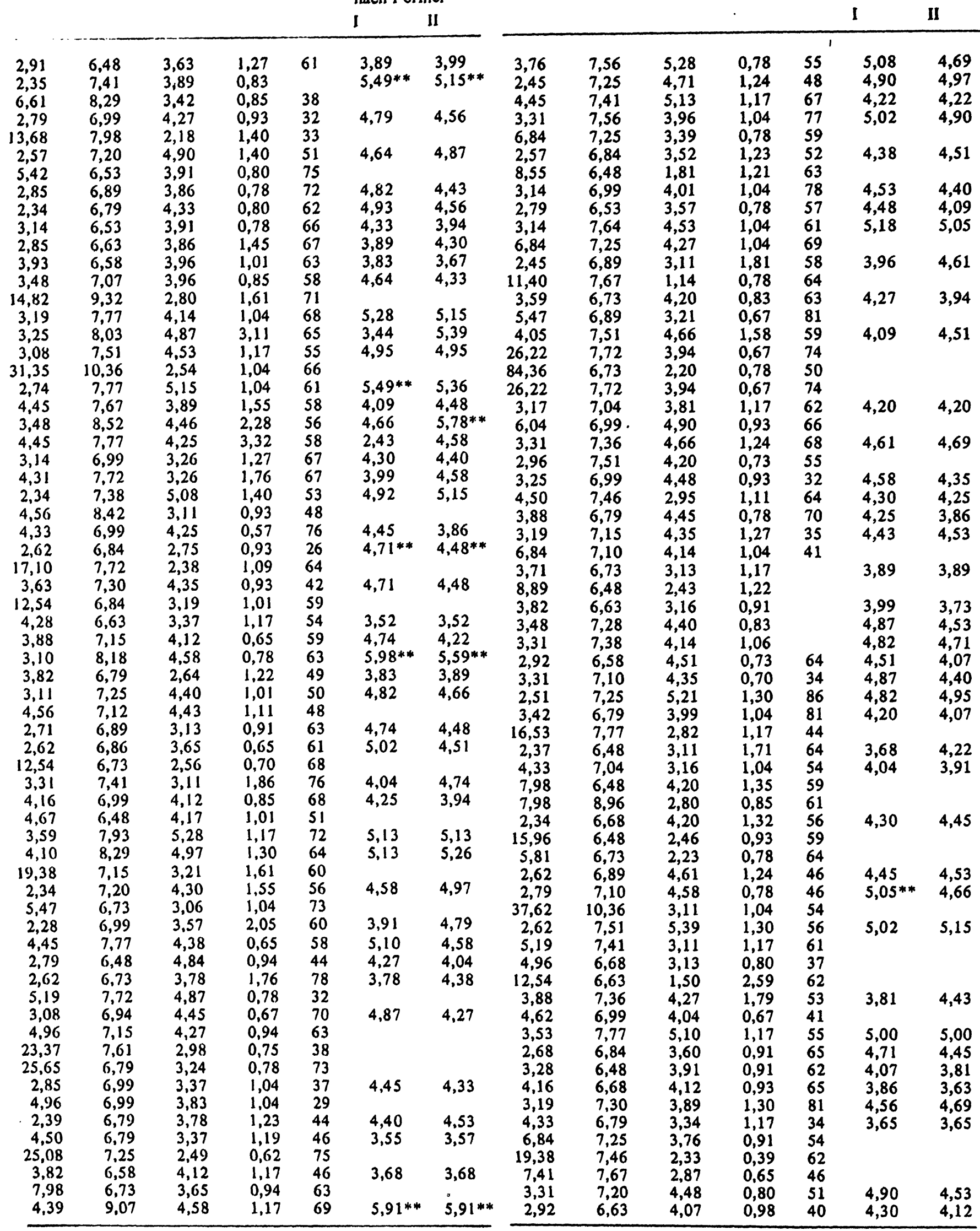


Typ IV

\begin{tabular}{|c|c|c|c|c|c|}
\hline $\begin{array}{l}\text { Trigly- } \\
\text { ceride }\end{array}$ & $\begin{array}{l}\text { Chole- } \\
\text { sterin }\end{array}$ & $\begin{array}{l}\beta \text {-Lipo- } \\
\text { protein- } \\
\text { chole- } \\
\text { sterin }\end{array}$ & $\begin{array}{l}\alpha \text {-Lipo- } \\
\text { protein- } \\
\text { chole- } \\
\text { sterin }\end{array}$ & Alter & $\begin{array}{l}\text { berechnetes } \\
\beta \text {-Lipoprotein- } \\
\text { cholesterin }\end{array}$ \\
\hline
\end{tabular}

$[\mathrm{mmol} /]$ [mmol/1] [mmol/1] [mmol/1] [Jahre] [mmol/1]

\begin{tabular}{rllllll} 
& & & & \multicolumn{3}{c}{ nach Formel } \\
& & & & \multicolumn{3}{c}{ I } \\
\hline 3,08 & 7,25 & 3,57 & 1.04 & 46 & 4,82 & 4,69 \\
2,45 & 6,48 & 4,45 & 1,14 & 70 & 4,22 & 4,20 \\
5,70 & 7,30 & 5,10 & 0,96 & 68 & & \\
7,98 & 6,73 & 1,63 & 0,88 & 42 & & \\
6,95 & 7,15 & 2,67 & 1,01 & 42 & & \\
39,90 & 6,48 & 2,59 & 1,17 & 40 & & \\
3,02 & 6,48 & 3,86 & 0,54 & 61 & 4,56 & 3,94 \\
57,00 & 8,39 & 2,85 & 0,65 & 61 & & \\
13,68 & 7,25 & 2,38 & 1,17 & 75 & & \\
4,16 & 6,88 & 3,24 & 0,65 & 37 & 4,25 & 3,73 \\
3,63 & 6,88 & 4,74 & 0,70 & 52 & 4,43 & 3,96 \\
2,39 & 6,53 & 2,84 & 2,33 & 49 & 3,11 & 4,27 \\
3,09 & 6,97 & 3,68 & 1,24 & 61 & 4,30 & 3,38 \\
4,67 & 7,51 & 4,61 & 1,04 & 45 & & \\
34,20 & 6,58 & 2,75 & 1,61 & 31 & & \\
2,74 & 6,53 & 3,26 & 0,67 & 47 & 4,61 & 4,12 \\
2,51 & 6,53 & 3,89 & 0,57 & 48 & 4,82 & 4,22 \\
\hline
\end{tabular}

\section{Literatur}

1. Lees, R. S. \& Hatch, F. T. (1963) J. Lab. Clin. Med. 61, 518-524.

2. Fredrickson, D. S., Levy, R. I. \& Lees, R. S. (1967) N. Engl. J. Med. 276, 32-44, 94-103, 148-156, 215-226, 273281.

3. Ahrens jr., E. H., Hirsch, J., Oette, K., Farquhar, J. W. \& Stein, Y. (1961) Trans. Ass. Amer. Phycns. 74, 134-146.

4. Gries, F. A., Jahnke, K., Preiss, H., Canzler, H. \& Miss, H. D. (1969) Deut. Med. Wochenschr. 94, 2307-2315.

5. Rapp, W. \& Kahlke, W. (1968) Clin. Chim. Acta 19, 493.

6. Noble, R. P. (1968) J. Lipid Res. 9, 693-700.

7. Greten, H., Seidel, D. \& Kolbe, J. (1970) Deut. Med. Wochenschr. 34, 1716-1723.

8. Beaumont, J. L., Carlson, L. A., Cooper, G. R., Fejfar, Z., Fredrickson, D. S. \& Strasser, I. (1970) Bull. Wld. Hlth. Org. 43, 891-915.
Typ IV

\begin{tabular}{lllll}
\hline $\begin{array}{l}\text { Trigly- } \\
\text { ceride }\end{array}$ & $\begin{array}{l}\text { Chole- } \\
\text { sterin }\end{array}$ & $\begin{array}{l}\beta \text {-Lipo- } \\
\text { protein- } \\
\text { cholc- } \\
\text { sterin }\end{array}$ & $\begin{array}{l}\alpha \text {-Lipo- } \\
\text { protein- } \\
\text { chole- }\end{array}$ & Alter \\
& & $\begin{array}{l}\text { berechnetes } \\
\beta \text {-Lipoprotein- } \\
\text { cholesterin }\end{array}$ &
\end{tabular}

[mmol/1] [mmol/l] [mmol/1] [mmol/l] [Jahre] [mmol/1]

\begin{tabular}{rrrrrrr} 
& & & & \multicolumn{3}{c}{ nach Formel } \\
& & & & \multicolumn{2}{c}{ I } & II \\
\hline 5,13 & 7,72 & 5,00 & 0,65 & & & \\
6,09 & 6,79 & 4,20 & 1,17 & 69 & 4,40 & 4,40 \\
3,14 & 6,99 & 4,66 & 0,98 & 43 & 4,58 & 4,40 \\
38,76 & 7,77 & 2,38 & 0,52 & 83 & & \\
5,13 & 7,56 & 3,96 & 0,93 & 41 & & \\
7,62 & 6,79 & 4,22 & 0,39 & 45 & & \\
6,01 & 6,68 & 3,24 & 0,96 & 62 & & \\
6,27 & 7,41 & 4,04 & 1,94 & 45 & & \\
14,82 & 8,55 & 4,48 & 0,75 & 59 & & \\
4,42 & 6,48 & 3,91 & 0,75 & & 3,70 & 3,29 \\
3,82 & 7,30 & 5,05 & 0,78 & 65 & 4,79 & 4,40 \\
3,28 & 6,73 & 3,89 & 1,04 & 53 & 4,20 & 4,07 \\
5,07 & 7,12 & 3,99 & 0,94 & 49 & & \\
5,70 & 6,84 & 4,43 & 0,78 & 49 & & \\
7,98 & 6,63 & 3,24 & 0,78 & 34 & & \\
3,25 & 6,79 & 4,20 & 1,17 & 52 & 4,14 & 4,14 \\
2,79 & 6,73 & 4,25 & 0,96 & 55 & 4,51 & 4,30 \\
\hline
\end{tabular}

9. Fredrickson, D. S. (1972) Modern Concepts of Cardiovascular Disease XLI: 31-36.

10. Seidel, D. \& Greten, H. (1970) Clin. Chim. Acta 30, 31-36.

11. Wieland, H. \& Seidel, D. (1973) Clin. Chem. 19, 1139-1141.

12. Kessler, G. \& Lederer, H. (1966) Technicon-Symposium über Automation in der analyt. Chemie, 1965 New York, p. 341 .

13. Seidel, D., Greten, H., Geisen, H. P., Wengeler, H. \& Wieland, H. (1972) Eur. J. Clin. Invest. 2, 359-364.

14. Friedewald, W. T., Levy, R. I. \& Fredrickson, D. S. (1972) Clin. Chem. 18, 499-502.

15. Schonfeld, G. (1970) J. Lab. Clin. Med. 75, 206-211.

16. Ruderman, N. B., Jones, A. L. Krauss, R. M. \& Shafrir, E. (1971) J. Clin. Invest. 50, 1355-1368.

17. Wilson, D. E. \& Spiger, M. J. (1973) J. Lab. Clin. Med. 82, 473-482.
Prof. Dr. G. Schlierf

Medizinische Universitätsklinik 6900 Heidelberg, Bergheimer Str. 58 
.

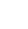

